\section{$\Rightarrow$ T-CELL ACTIVATION}

HS1 functions as an essential actin-regulatory adaptor protein at the immune synapse.

Gomez, T. S. et al. Immunity 24, 741-752 (2006)

Formation of the immunological synapse, which involves actin polymerization and recruitment of signalling molecules, is crucial for T-cell activation. Now, new research has implicated haematopoietic-lineage-cell-specific protein 1 (HS1) as a key regulatory adaptor protein in this process. Using T cells from $\mathrm{Hs}^{-1-}$ mice and T cells in which the expression of HS1 is suppressed, the authors showed that HS1 maintains F-actin at the immunological synapse and is required for the organization and maintenance of specific structures that are necessary for conjugate formation. Phosphorylation of HS1, by key signalling molecules in the synapse, is required for actin regulation and is important for the activation of interleukin-2 promoter. This protein is also important for maintaining VAV1, which regulates actin responses, at the synapse. So, HS1 is an actin-regulatory protein that is crucial for T-cell activation.

\section{EVOLUTION}

Insect immunity shows specificity in protection upon secondary pathogen exposure.

Sadd, B. M. \& Schmid-Hempel, P. Curr. Biol. 16, 1206-1210 (2006)

Immunological memory and specificity characterizes adaptive immunity in vertebrates. Invertebrates can mount an immune response following pathogen exposure, but it was not clear whether this response could be specific. A new study now shows that insects can mount a specific immune response to a previously encountered pathogen. Sadd and Schmid-Hempel tested the immune system of Bombus terrestris (the bumblebee) to determine if it could show protection, specificity or both on exposure to bacterial pathogens following previous exposure. The authors found that the immune system of B. terrestris responded in a specific manner to previously encountered pathogens, even several weeks after the first exposure, and that this response was functionally protective. So, despite their broad differences, both vertebrate and invertebrate immune systems mount a specific protective response. The authors suggest that similar selective pressures on these different immune systems over evolutionary time might therefore have resulted in comparable solutions.

\section{APOPTOSIS}

\section{Gimap4 accelerates T-cell death.}

Schnell, S. et al. Blood 108, 591-599 (2006)

By searching for genes that are differentially expressed during T-cell development, the authors of this study identified Gimap4 (GTPase, immunity-associated protein family member 4).

Gimap4 expression was found to be induced initially during the early stages of T-cell development, turned off during positive selection and re-expressed thereafter, including by mature T cells. Despite this expression pattern, Gimap $4^{-/-}$mice showed no defect in T-cell development. Instead, the authors found that, compared with wild-type cells, cultures of mature T cells from Gimap $4^{-/}$mice showed increased numbers of apoptotic cells versus dead cells following serum starvation or exposure to other apoptotic triggers. Further studies led the authors to conclude that GIMAP4 acts as an accelerator of the late stages of apoptosis and functions downstream of caspase- 3 activation and phosphatidylserine exposure.

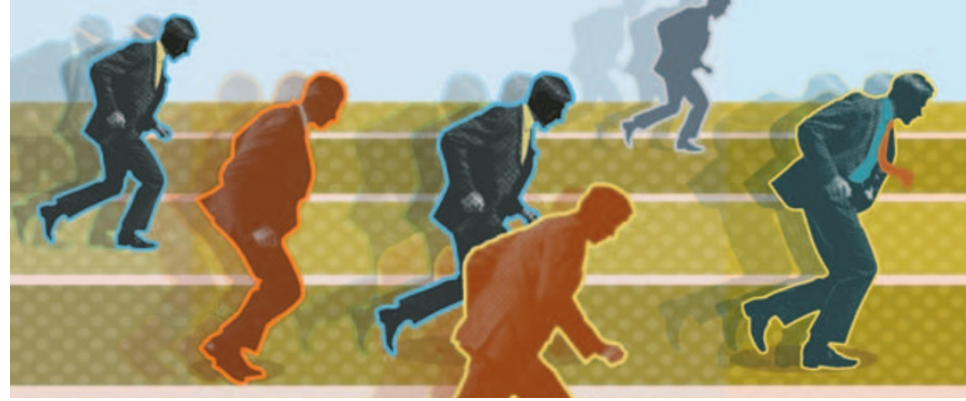

REGULATORY T CELLS

\title{
In hot pursuit
}

Despite having been identified more than 10 years ago, $\mathrm{CD} 4^{+} \mathrm{CD} 25^{+}$ regulatory $\mathrm{T}\left(\mathrm{T}_{\mathrm{Reg}}\right)$ cells are still being characterized. Naturally occurring $\mathrm{T}_{\mathrm{Reg}}$ cells express the transcription factor forkhead box P3 (FOXP3), which has been identified as a major marker and regulator of the development and function of $\mathrm{T}_{\mathrm{Reg}}$ cells. However, identifying and isolating human $\mathrm{T}_{\text {Reg }}$ cells for use in functional studies is difficult, because these cells cannot easily be distinguished from activated $\mathrm{CD}^{+}$non-regulatory T cells. Also, as FOXP3 is an intracellular protein, fixation and permeabilization of the cells is required. Two papers in The Journal of Experimental Medicine now report that low surface expression of CD127 (the $\alpha$-chain of the IL-7 receptor) can be used effectively to identify human $\mathrm{T}_{\text {Reg }}$ cells.

In an effort to identify new cellsurface markers for human $\mathrm{T}_{\mathrm{Reg}}$ cells, Liu et al. used gene-expression studies to compare two subsets of human T cells: $\mathrm{CD} 4^{+} \mathrm{CD} 25^{\text {hi }} \mathrm{T}$ cells and CD $4^{+} \mathrm{CD} 25^{-} \mathrm{T}$ cells. They noted in particular that expression of $C D 127$ mRNA was inversely correlated with expression of CD25. Subsequent analyses showed that most of the FOXP3 ${ }^{+}$ $\mathrm{T}$ cells were in the CD127 $7^{\text {low/- }} \mathrm{T}$-cell subset and, further, that the highest levels of FOXP3 expression correlated with the lowest levels of CD127 expression. Using multi-parameter flow cytometry, the authors determined that a broad gating strategy of $\mathrm{CD} 4{ }^{+} \mathrm{CD} 25^{+} \mathrm{CD} 127^{\text {low/- }}$ accounted for $\sim 80 \%$ of the FOXP3-expressing $\mathrm{T}$ cells. Up to $10 \%$ of all the $\mathrm{CD} 4^{+} \mathrm{T}$ cells were identified as potential $\mathrm{T}_{\mathrm{Reg}}$ cells - a considerably higher percentage than has been shown previously. Liu et al. also found that CD127 could be used, independently of CD25, to separate a suppressive T-cell subset, which could be useful in future therapeutic applications. The authors concluded that using a combination of CD4, CD25 and CD127 results in highly purified $\mathrm{T}_{\mathrm{Reg}}$-cell population.

In the second paper, Seddiki et al. reasoned that because of the IL-7dependent and IL-2-independent nature of many non- $\mathrm{T}_{\mathrm{Reg}}$-cell subsets, $\mathrm{T}_{\text {Reg }}$ cells (which require IL-2) might not require IL-7. Using monoclonal antibodies specific for CD4, CD25 (the $\alpha$-chain of the IL-2 receptor) and CD127, they found that CD127 expression could be used to distinguish two populations of $\mathrm{CD} 4^{+} \mathrm{CD} 25^{+}$ T cells - a small CD $25^{+} \mathrm{CD} 127^{\text {low }}$ population and a much larger $\mathrm{CD} 25^{+} \mathrm{CD} 127^{\mathrm{hi}}$ population. They noted a strong correlation between the number of $\mathrm{CD} 25^{+} \mathrm{CD} 127^{\text {low }}$ $\mathrm{T}$ cells and $\mathrm{CD} 25^{+} \mathrm{FOXP}^{+} \mathrm{T}$ cells. Interestingly, Seddiki et al. saw this correlation in cells that were gated on either CD45RA- or CD45RA+ populations, indicating that $\mathrm{CD} 25^{+} \mathrm{CD} 127^{\text {low }}$ $\mathrm{T}_{\mathrm{Reg}}$ cells can be found within both naive and antigen-experienced T-cell subsets. Finally, the authors looked at the in vitro suppressive activity of the $\mathrm{T}_{\text {Reg }}$-cell population and found that it was restricted to the $\mathrm{CD} 25^{+} \mathrm{CD} 127^{\text {low }}$ T cells.

Together, these two reports show that human $\mathrm{T}_{\mathrm{Reg}}$ cells can be identified by their low cell-surface expression of CD127. The ability to selectively enrich these cells for in vitro functional studies will hopefully lead to a greater understanding of how they contribute to the immunopathology of disease, and increase the potential of these cells for in vivo therapy.

Sharon Ahmad

ORIGINAL RESEARCH PAPERS Liu, W. et al. $\mathrm{CD} 127$ expression inversely correlates with FoxP3 and suppressive function of human $\mathrm{CD}^{+} \mathrm{T}$ reg cells. J. Exp. Med. 203, 1701-1711(2006) | Seddiki, N. et al. Expression of interleukin (IL)-2 and IL-7 receptors discriminates between human regulatory and activated T cells.J. Exp. Med. 203, 1693-1700 (2006) 